\title{
Supervised consumption services for acute care hospital patients
}

\author{
Kathryn A. Dong MD MSc, Jennifer Brouwer RN MN, Curtis Johnston MD, Elaine Hyshka PhD
}

Cite as: CMAJ 2020 May 4;192:E476-9. doi: 10.1503/cmaj.191365

A ccess to supervised consumption services is considered part of the standard of care for people with opioid use disorder and is recommended across the treatment continuum. ${ }^{1}$ People who use substances require acute care services more frequently, and past research indicates that many will continue to use substances in hospital. ${ }^{2}$ Written or unwritten expectations of abstinence, stigma and untreated pain or withdrawal may result in patients selfmedicating with illegal substances, taking measures to conceal drug use (most commonly by using in a locked hospital washroom), leaving hospital for prolonged periods of time or being involuntarily discharged. ${ }^{3}$

Supervised consumption services provide hygienic environments in which people consume illegal substances under the observation of staff trained in overdose prevention and response, without the risk of arrest for drug possession. ${ }^{4} \mathrm{Peo}-$ ple who use substances have identified the integration of supervised consumption services into acute care hospitals as a key strategy for facilitating completion of medical treatment and promoting patient-centred care. ${ }^{5}$ Canadian cohort data suggest that $68.2 \%$ of people who inject drugs would be willing to access a supervised consumption service for inpatients. ${ }^{6}$ Medical experts have identified the embedding of supervised consumption services into acute care hospitals as a key next step. ${ }^{4}$

\section{What is an acute care supervised consumption service?}

Supervised consumption services in acute care provide a safe, secure environment in which hospital patients can use preobtained illegal substances and be monitored by staff. As with community-based supervised consumption services, education on safer use, provision and disposal of sterile equipment, and access to immediate assistance in the case of an adverse reaction are provided. Ideally, patients are also able to access evidence-based treatment for their substance use disorder(s) and additional health and social supports via referral to an associated consult service or provider.

For patients who do not wish to use the supervised consumption service but intend to consume substances while in hospital,

\section{KEY POINTS}

- Access to supervised consumption services is recommended across the treatment continuum for people with opioid use disorder.

- The hospital environment can place patients with ongoing substance use at risk.

- Embedded supervised consumption services in acute care hospitals have the potential to improve safety and engage patients in addiction treatment.

these services may also provide sterile supplies for drug consumption, personal sharps containers, and overdose prevention education and naloxone kits, alongside ongoing efforts to engage patients into care within the service.

To our knowledge, worldwide there are 5 supervised consumption services based in acute care hospitals. Three are in Europe -1 is located in an outpatient addiction treatment program within a hospital in Barcelona ${ }^{7}$ and 2 others are located within, but administratively separate from, hospitals in Paris and Strasbourg in France; these are designed for community, not patient, access. ${ }^{8}$ In early 2018, the Royal Alexandra Hospital in Edmonton, Alberta, opened the first supervised consumption service for inpatients in North America (Box 1). Shortly after, an overdose prevention site was established on the grounds of St. Paul's Hospital in Vancouver. ${ }^{9}$ Additionally, the Dr. Peter Centre, an integrated HIV/AIDS outpatient and residential care facility, is located close to St. Paul's Hospital and has been offering supervised consumption services to its registered clients, including those admitted to hospital, since 2002.

\section{How can supervised consumption services be delivered to patients in acute care hospitals?}

Supervised consumption service models in acute care should reflect the local needs of patients and hospital staff. A designated location embedded within the hospital, staffed by a core group of providers with extra training in substance use disorders and harm reduction, may facilitate trust and uptake 
Box 1: Supervised consumption services at the Royal Alexandra Hospital, Edmonton, Alberta

The supervised consumption service at the Royal Alexandra Hospital is open daily except for $1130-1230$ and $1800-1900$, during scheduled staff breaks. Upon presenting to the supervised consumption service, individuals are double identified (i.e., name and birthdate) and then confirmed to be a hospital patient. An initial assessment is completed in an intake area to assess medical stability, substances to be consumed (self-report) and route of use. Patients sign written informed consent and complete a patient agreement before using the service. Safer use education and sterile supplies are provided as required. Patients then consume their pre-obtained illegal substances orally, nasally or via injection, in 1 of 4 consumption booths (Figure 1).

After use, patients are encouraged to stay for a 20-minute monitoring period to ensure no adverse reactions have occurred (Figure 2). As the hospital does not have an electronic medical record, patients' care teams are notified of access by a Report of Supervised Consumption Service Access form that is faxed to the unit and placed in the patient chart. All patients have access to an addiction medicine consult team, which provides immediate access to all forms of opioid agonist treatment, psychosocial support and treatment referrals, and interventions for social stabilization and targeted health promotion. The addiction medicine clinician who completes the initial consult often makes a written suggestion in the patient care orders that the patient should have access to the supervised consumption service. However, some patients are identified as needing access to the consult team through presentation to the supervised consumption service.

Between Apr. 2, 2018, and Nov. 30, 2019, there were 7856 visits to the consumption room by 199 unique patients. This included 3165 visits for injectable opioid agonist treatment, which was integrated into the service on Oct. 15, 2018 , and 35 visits by emergency department patients who were eligible for access starting Oct. 1, 2019.

Staff responded to 27 emergency situations in the service; there were no deaths. Seventy patients were identified as being in need of referral to the addiction medicine consult service when they self-presented to the supervised consumption service. Opioids were the most commonly used substances ( $87 \%$ of total substances consumed, including injectable opioid agonist treatment); $13 \%$ of substances consumed were stimulants. Most substances were consumed via intravenous injection (96\%); patients used their intravenous catheters in 5\% of these cases.

among patients. Alternatively, a bedside model of supervision, such as available to residents at the Dr. Peter Centre in Vancouver, may provide greater access to care, particularly for patients with severe health or mobility issues. Residents of the Dr. Peter Centre who are admitted to St. Paul's Hospital may travel across the street to the supervised consumption space embedded in the centre.

Addressing existing hospital culture is important and may be a greater challenge than securing funding and implementing the service. ${ }^{9,10}$ Cultivating a philosophy of harm reduction, reducing stigma by creating a greater understanding of substance use disorders and engaging staff in addressing the harms associated with ongoing drug use in hospital are also important. Furthermore, it is critical to have a strong, clear and visible commitment by hospital leadership, as well as a local champion. ${ }^{9}$ Ongoing site-wide education and a mechanism for identifying and addressing staff concerns can help to facilitate change.

Supervised consumption services must obtain an exemption from Health Canada under section 56.1 of the Controlled Drugs and Substances Act; temporary overdose prevention sites can be approved and set up more quickly via other regulatory pathways. Patients bring their own pre-obtained substances to consume.

\section{Who is eligible to access these services?}

These services are designed to improve the care and safety of hospital inpatients, but several other groups should also be considered for access. These include patients in the emergency department, outpatients accessing other services on hospital property and hospital visitors. One supervised consumption service in Barcelona and the overdose prevention site located on the St. Paul's Hospital campus in Vancouver provide services to the public as well as hospital patients (outpatients and inpatients). The overdose prevention site at St. Paul's Hospital is located in the basement and operates 12 hours per day; about $20 \%$ of visits are from hospital inpatients. ${ }^{9}$ Depending on location and the availability of other services, hospital campuses may be feasible settings for supervised consumption services that serve an entire community.

\section{What are the possible harms?}

Few harms associated with supervised consumption services are identified in the scientific literature, and evidence shows that overdose events are successfully managed in these facilities on a daily basis. ${ }^{11,12}$ However, as patients in hospital are less medically stable than individuals presenting to a supervised consumption service in the community, adverse events associated with substance use may be more difficult to manage. Because of ongoing stigmatization of substance use disorders in Canadian society, some hospital staff may feel moral distress related to implementing a supervised consumption service in a hospital setting; care should be taken to ensure such concerns are managed in a way that ensures optimal patient care as well as addresses the needs of the staff in a supportive manner. In addition, patients may be concerned that accessing the supervised consumption service will result in discrimination, stigmatization or changes to their medications, particularly prescribed opioids. ${ }^{5}$

\section{What is the evidence so far?}

In general, supervised consumption services have been found to promote safer injecting conditions, reduce the number of fatal overdoses and improve access to other health care and addiction 


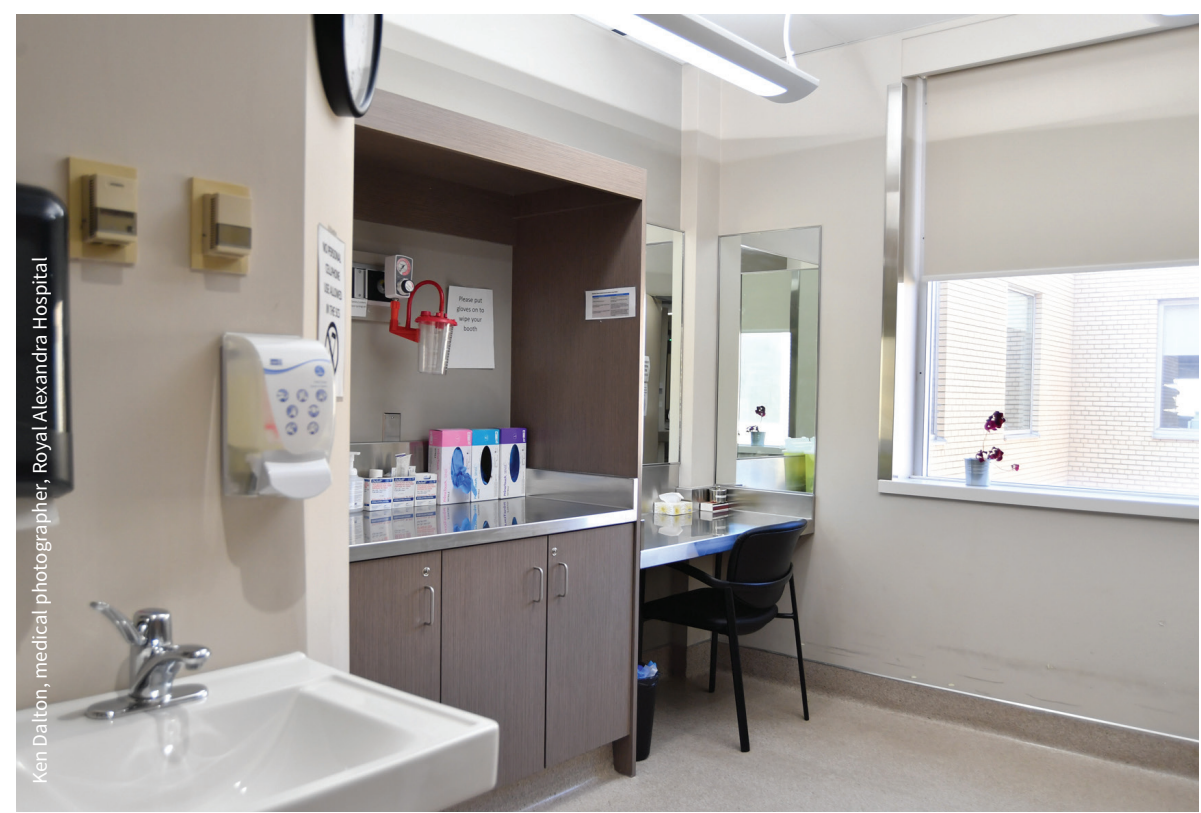

Figure 1: Consumption booth in the supervised consumption service at the Royal Alexandra Hospital, Edmonton, Alberta.

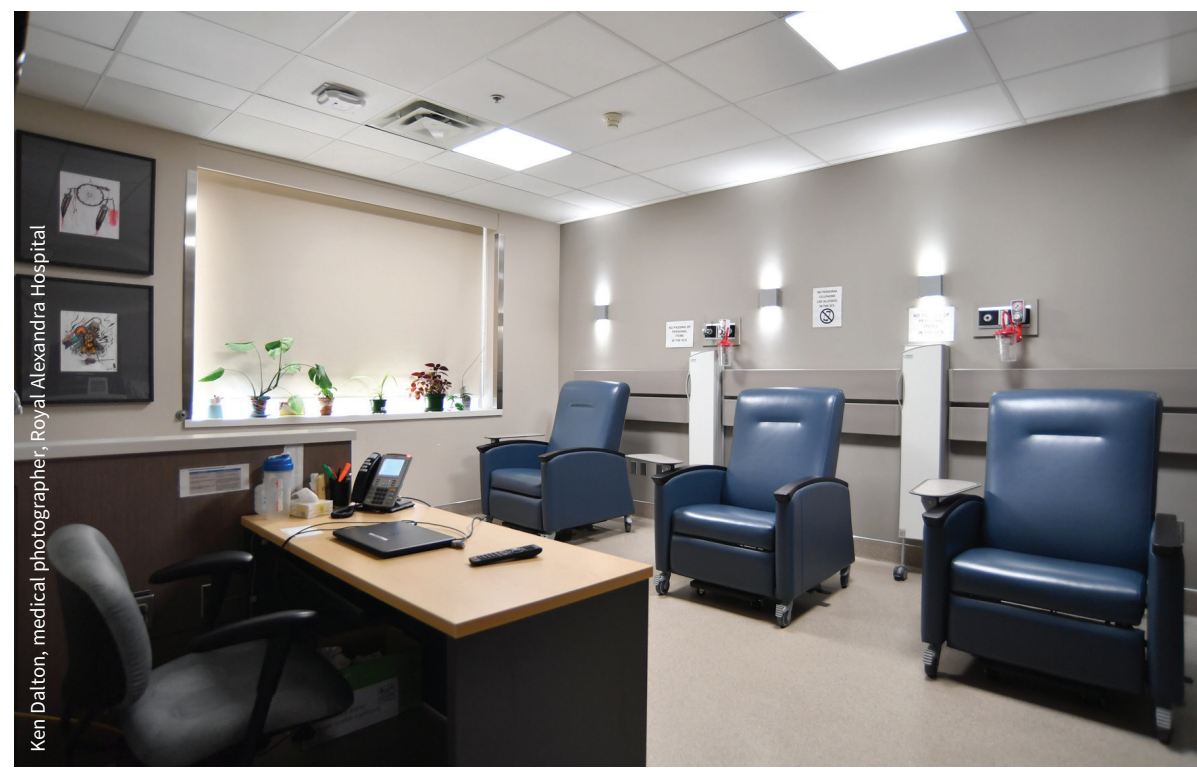

Figure 2: Post-use observation area in the supervised consumption service at the Royal Alexandra Hospital, Edmonton, Alberta. the numbers of the following: fatal and near-fatal opioid poisonings on hospital property, improperly discarded syringes on hospital property, peripherally and centrally inserted catheters that need to be replaced owing to suspected tampering and drug use, and people who use substances leaving against medical advice and who subsequently require readmission.

\section{What can be expected in the future?}

As the harms associated with the opioid crisis continue, hospital providers will be expected to identify patients with substance use disorders, begin evidence-based treatment and ensure patients are not at an increased risk of death or infection while in their care. Embedded supervised consumption services within acute care hospitals may help to achieve these goals and should be considered for widespread expansion, most urgently to those facilities on the grounds of which unintentional, unwitnessed opioid overdoses are already occurring. Provision of supervised inhalation and prescribed medications for self-administration as an alternative to illegally manufactured and potentially toxic substances should also be considered. Further research to document patient and staff perspectives, health benefits, uptake into substance use treatment, costeffectiveness and any unanticipated adverse effects of acute care supervised consumption services will facilitate planning and implementation at other sites. services. ${ }^{11}$ They are cost-effective and have been associated with improvements in public order (e.g., a reduction in publicly discarded syringes) without increasing drug-related crime. ${ }^{12}$ One recent study found that participants in the programs at the Dr. Peter Centre were less likely than other HIV-positive people who use drugs to leave St. Paul's Hospital against medical advice when in hospital. ${ }^{13}$ The authors hypothesized that the nearby access to supervised consumption services at the centre was one factor supporting increased likelihood of care completion. ${ }^{13}$

Other potential benefits to acute care patients that warrant further study include improved patient hospital experience ${ }^{7}$ and improved identification of people who require treatment and support for substance use disorders, along with reductions in

\section{References}

1. Bruneau J, Ahamad K, Goyer M, et al. Management of opioid use disorders: a national clinical practice guideline. CMAJ 2018;190:E247-57.

2. Grewal HK, Ti L, Hayashi K, et al. Illicit drug use in acute care settings. Drug Alcohol Rev 2015;34:499-502.

3. McNeil R, Small W, Wood E, et al. Hospitals as a 'risk environment': an ethnoepidemiological study of voluntary and involuntary discharge from hospital against medical advice among people who inject drugs. Soc Sci Med 2014;105:59-66.

4. Supervised consumption services: operational guidance. Vancouver: British Columbia Centre on Substance Use; 2017. Available: www.bccsu.ca/wp-content /uploads/2017/07/BC-SCS-Operational-Guidance.pdf (accessed 2019 Oct. 11).

5. McNeil R, Kerr T, Pauly B, et al. Advancing patient-centered care for structurally vulnerable drug using populations: a qualitative study of the perspectives of people who use drugs regarding the potential integration of harm reduction interventions into hospitals. Addiction 2016;111:685-94. 
6. Ti L, Buxton J, Harrison S, et al. Willingness to access an in-hospital supervised injection facility among hospitalized people who use illicit drugs. J Hosp Med 2015;10:301-6.

7. Daigre C, Comin M, Rodriguez-Cintas L, et al. Users' perception of a harm reduction program in an outpatient drug dependency treatment center. Gac Sanit 2010;24:446-52.

8. Jauffret-Roustide M. Les salles de consummation à moindre risque: apprendre à vivre avec les drogues. Esprit. 2016 Novembre; n 429: 115-123.

9. Overdose Prevention Site at St. Paul's Hospital. Toronto: Canadian AIDS Treatment Information Exchange; 2019. Available: www.catie.ca/en/pc/program/ ops-stpaul (accessed 2020 Jan. 4).
10. Hyshka $\mathrm{E}$, Morris $\mathrm{H}$, Anderson-Baron J, et al. Patient perspectives on a harm reduction-oriented addiction medicine consultation team implemented in a large acute care hospital. Drug Alcohol Depend 2019;204:107523.

11. Potier C, Laprévote V, Dubois-Arber F, et al. Supervised injection services: What has been demonstrated? A systematic literature review. Drug Alcohol Depend 2014;145:48-68.

12. Kennedy MC, Karamouzian M, Kerr T. Public health and public order outcomes associated with supervised drug consumption facilities: a systematic review. Curr HIV/AIDS Rep 2017;14:161-83.

13. Ti L, Milloy M-J, Turje RB, et al. The impact of an HIV/AIDS adult integrated health program on leaving hospital against medical advice among HIV-positive people who use illicit drugs. J Public Health (Oxf) 2017;39:e33-9.
Competing interests: Kathryn Dong reports receiving a grant from Alberta Health, for start-up and operating costs of the supervised consumption services at the Royal Alexandra Hospital. This grant was received during the conduct of this study, but none of these funds paid for Dr. Dong's time to write this article. Dr. Dong also receives a medical leadership salary from Alberta Health Services for her roles as Medical Director, Inner City Health and Wellness Program, during the conduct of the study. Jennifer Brouwer received a salary from Alberta Health Services for her role as Program Manager, Professional Practice Office and Inner City Health and Wellness, during the conduct of the study. Curtis Johnston received a medical leadership salary from Alberta Health Services for his role as Facility Medical Director, Royal Alexandra Hospital, and Associate Zone Medical Direc- tor, Edmonton Zone. Elaine Hyshka reports receiving grants from the Canadian Institutes of Health Research, partial salary support paid to her institution from the Royal Alexandra Hospital Foundation, during the conduct of the study; and partial salary support paid to her institution from Alberta Health Services, outside the submitted work Since the writing of this article, Ms. Brouwer has relocated to a different province and Dr. Johnston has moved to a different administrative position. These were their positions at the time of writing.

This article has been peer reviewed.

Affiliations: Department of Emergency Medicine (Dong), University of Alberta; Inner City Health and Wellness Program (Dong, Brouwer, Hyshka, Johnston), Royal Alexandra Hospital; Department of Critical Care Medicine (Johnston) and School of Public
Health (Hyshka), University of Alberta, Edmonton, Alta.

Contributors: All of the authors contributed to the conception and design of the work, drafted the manuscSript, revised it critically for important intellectual content, gave final approval of the version to be published and agreed to be accountable for all aspects of the work.

Funding: The supervised consumption service at the Royal Alexandra Hospital is funded by a grant from Alberta Health.

Acknowledgement: The authors thank Ms. Klaudia Dmitrienko for compiling the data for the Royal Alexandra Hospital supervised consumption service.

Correspondence to: Kathryn Dong, kathryni@ualberta.ca 